\title{
Inserción laboral de los graduados en Información y Documentación cinco años después de titularse
}

Employability of graduates in Library and Information
Science five years after graduation Ángel Borrego; Maite Comalat

Borrego, Ángel; Comalat, Maite (2021). "Inserción laboral de los graduados en Información y Documentación cinco años después de titularse". Anuario ThinkEPI, v. 15, e15a02.

https://doi.org/10.3145/thinkepi.2021.e15a02

Publicado en IweTel el 20 de octubre de 2021

\section{Ángel Borrego}

https://orcid.org/0000-0002-6462-3966

Universitat de Barcelona

Facultat d'Informació i Mitjans Audiovisuals

Centre de Recerca en Inf., Com. i Cultura (CRICC)

Melcior de Palau, 140. 08014, Barcelona (España)

borrego@ub.edu

\author{
Maite Comalat \\ https://orcid.org/0000-0002-4214-3320 \\ Universitat de Barcelona \\ Facultat d'Informació i Mitjans Audiovisuals \\ Centre de Recerca en Inf., Com. i Cultura (CRICC) \\ Melcior de Palau, 140. 08014, Barcelona (España) \\ comalat@ub.edu
}
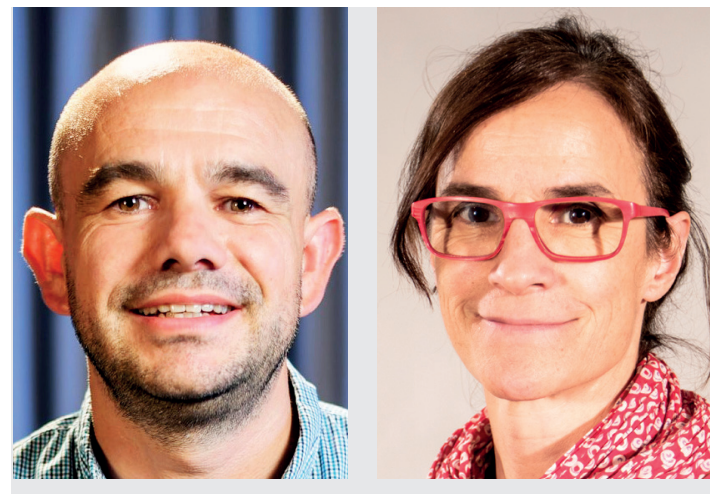

Resumen: Se sintetizan los resultados relativos a los graduados en Información y Documentación recogidos en la Encuesta de inserción laboral de titulados universitarios 2019 del Instituto Nacional de Estadística. Los datos indican que los graduados en Información y Documentación trabajan en una proporción ligeramente inferior al resto de titulados. Sin embargo, disfrutan de una mayor estabilidad laboral, con un porcentaje superior de contratos permanentes en detrimento de los contratos en prácticas y becas. Más de la cuarta parte de los graduados trabaja a tiempo parcial y la Administración Pública continúa siendo el ámbito que proporciona mayores oportunidades de ocupación. Se observa un cierto grado de sobrecualificación, con porcentajes significativos de graduados que afirman estar ocupando puestos de trabajo para los que no es necesaria la titulación. Las retribuciones son inferiores a las de otros graduados, al tiempo que se constata la misma brecha salarial de género que castiga a las tituladas de todas las disciplinas. Los graduados en Información y Documentación se declaran satisfechos con la titulación cursada y mayoritariamente repetirían los estudios, se muestran poco proclives a la movilidad internacional durante sus estudios y se consideran usuarios avanzados de las tecnologías de la información y la comunicación.

Palabras clave: Datos estadísticos; Educación; Empleabilidad; Inserción laboral; Mercado laboral.

Abstract: This article summarizes results related to graduates in Library and Information Science (LIS) provided in a report on higher-education employability published by the Spanish National Statistics Institute (INE) in 2019. The data show that LIS graduates have a slightly lower employment rate than other graduates. Nevertheless, they enjoy greater job stability, with a higher percentage of permanent contracts instead of placements or internships. More than one-quarter of LIS graduates work part-time, and the Public Administration continues to be the sector providing more employment opportunities for them. A certain level of overqualification is observed, with significant percentages of LIS graduates stating that they hold positions for which their degree is not necessary. Salaries are lower than those of other graduates, with data showing the same gender pay gap that affects female graduates across all disciplines. LIS graduates 
are satisfied with their training, and most would study the same degree again. They are little inclined to international mobility during their studies and consider themselves advanced users of information and communication technologies.

Keywords: Education; Employability; Job market; Labour market; Statistics.

\section{Introducción}

En octubre de 2020, el Instituto Nacional de Estadística presentó los resultados de la segunda edición de la Encuesta de inserción laboral de titulados universitarios 2019 (INE, 2020). Se trata de un estudio que "pretende investigar la transición de los titulados al mercado de trabajo: obtener datos y referentes sobre la calidad de la inserción laboral de la población titulada en las universidades españolas, conocer las condiciones con las que acceden al mercado laboral, la adecuación de los estudios al trabajo que realizan, el tipo de contrato o la movilidad. En definitiva, un conjunto de indicadores que permitan conocer la situación de este colectivo y relacionarla con la titulación que hayan cursado" (INE, 2020).

Se considera que, desde el momento de la titulación, son necesarios alrededor de tres años para estabilizar la situación laboral. Por esta razón, en el trabajo de campo realizado en 2019 para elaborar esta edición de la encuesta, se tomó como grupo de estudio a los titulados del curso 2013-2014. El tamaño de la muestra se fijó en aproximadamente 32.000 graduados universitarios y 12.000 titulados de máster. La primera edición de la encuesta, realizada en 2014, había estudiado a los graduados en el curso 2009-2010. En un artículo previo, Moreiro-González (2015) analizó los resultados de aquel estudio en conjunción con otras fuentes de datos sobre la inserción laboral de los profesionales de la información.

El objetivo de esta nota es resumir los principales resultados relativos a la inserción laboral de los graduados en Información y Documentación en esta segunda edición de la encuesta. Si bien el INE también ofrece información sobre los titulados en másteres, al no existir un catálogo cerrado de títulos, el análisis se refiere a grandes ramas de conocimiento sin resultados desglosados por titulación.

Las variables analizadas hacen referencia a cuatro aspectos:

- nivel de formación de los titulados;

proceso de incorporación al mercado laboral;

idoneidad de los conocimientos adquiridos;

aptitudes personales para el trabajo desarrollado.

Para contextualizar los datos, las cifras correspondientes a los graduados en Información y Documentación se ofrecen junto a las globales de "Ciencias sociales, periodismo y documentación", categoría en la que están incluidos estos estudios.

Los resultados del estudio del INE hacen referencia a los 484 graduados en Información y Documentación en el curso 2013-2014 (tabla 1). De ellos, 470 residían en España en el momento de realización de la encuesta en 2019. Las 14 restantes, todas mujeres, vivían en el Reino Unido. La distribución de graduados por género no resulta sorprendente si tenemos en cuenta el perfil de los estudiantes de la titulación (De-la-Moneda, 2018), en buena parte perpetuado por una imagen de la profesión altamente feminizada.

Tabla 1. Graduados universitarios en el curso 2013-2014.

\begin{tabular}{|c|c|c|c|c|c|}
\hline & \multirow{2}{*}{\multicolumn{2}{|c|}{ Hombres }} & & & \\
\hline & & & \multicolumn{2}{|c|}{ Mujeres } & \multirow{2}{*}{$\begin{array}{c}\text { Total } \\
\mathbf{n}\end{array}$} \\
\hline & $\mathbf{n}$ & $\%$ & $\mathbf{n}$ & $\%$ & \\
\hline Información y Documentación & 142 & $29 \%$ & 342 & $71 \%$ & 484 \\
\hline Ciencias Sociales, Periodismo y Documentación & 7.734 & $36 \%$ & 13.507 & $64 \%$ & 21.241 \\
\hline Total & 96.857 & $41 \%$ & 136.769 & $59 \%$ & 233.626 \\
\hline
\end{tabular}

\section{Situación laboral en 2019}

Los resultados de la tabla 2 ponen de manifiesto que los graduados en Información y Documentación trabajan en una proporción ligeramente inferior a la del resto de graduados universitarios. Sin embargo, entre aquellos que trabajan (tabla 3), se observa una mayor estabilidad laboral, con más contratos permanentes y menos contratos en prácticas y becas. Por otro lado, los graduados en Información y Documentación presentan una menor iniciativa empresarial a la luz de los porcentajes de trabajadores independientes y empresarios. 
Tabla 2. Situación laboral en 2019 de los graduados universitarios en el curso 2013-2014.

\begin{tabular}{|c|c|c|c|c|c|c|c|}
\hline & \multicolumn{2}{|c|}{ Trabajando } & \multicolumn{2}{|c|}{ En desempleo } & \multicolumn{2}{|c|}{ Inactivo } & \multirow{2}{*}{$\begin{array}{c}\text { Total } \\
\mathbf{N}\end{array}$} \\
\hline & $\mathbf{n}$ & $\%$ & $\mathbf{n}$ & $\%$ & $\mathbf{n}$ & $\%$ & \\
\hline Información y Documentación & 401 & 82,8 & 66 & 13,6 & 18 & 3,6 & 484 \\
\hline Ciencias Sociales, Periodismo y Documentación & 18.081 & 85,1 & 1.756 & 8,3 & 1.403 & 6,6 & 21.241 \\
\hline Total & 201.138 & 86,1 & 17.588 & 7,5 & 14.901 & 6,4 & 233.626 \\
\hline
\end{tabular}

Tabla 3. Situación laboral en 2019 de los graduados universitarios del curso 2013-2014 que trabajaban en el momento de realización de la encuesta (\%).

\begin{tabular}{|l|c|c|c|c|c|c|}
\cline { 2 - 6 } & $\begin{array}{c}\text { En prácticas, } \\
\text { formación o } \\
\text { becario }\end{array}$ & $\begin{array}{c}\text { Asalariado } \\
\text { con contrato } \\
\text { permanente }\end{array}$ & $\begin{array}{c}\text { Asalariado } \\
\text { con contrato } \\
\text { temporal }\end{array}$ & $\begin{array}{c}\text { Empresario } \\
\text { con asalaria- } \\
\text { dos }\end{array}$ & $\begin{array}{c}\text { Trabajador inde- } \\
\text { pendiente o } \\
\text { empresario sin } \\
\text { asalariados }\end{array}$ & $\begin{array}{c}\text { Ayuda } \\
\text { familiar }\end{array}$ \\
\hline $\begin{array}{l}\text { Información y } \\
\text { Documentación }\end{array}$ & 0,8 & 67,0 & 24,4 & 0,5 & 7,3 & 0,0 \\
\hline $\begin{array}{l}\text { Ciencias Sociales, } \\
\text { Periodismo y } \\
\text { Documentación }\end{array}$ & 5,0 & 58,3 & 25,1 & 1,0 & 9,9 & 0,7 \\
\hline Total & 4,8 & 58,9 & 27,0 & 1,9 & 6,8 & 0,6 \\
\hline
\end{tabular}

Más de la cuarta parte de los graduados en Información y Documentación (28\%) trabajan a tiempo parcial (tabla 4), un porcentaje que supera en más de 11 puntos al del conjunto de graduados en Ciencias Sociales y que se sitúa 14 puntos por encima del global para el total de titulados.

Tabla 4. Jornada laboral en 2019 de los graduados universitarios del curso 2013-2014 que trabajaban en el momento de realización de la encuesta $(\%)$.

\begin{tabular}{|l|c|c|}
\cline { 2 - 3 } \multicolumn{1}{c|}{} & A tiempo completo & A tiempo parcial \\
\hline Información y Documentación & 71,6 & 28,4 \\
\hline Ciencias Sociales, Periodismo y Documentación & 83,0 & 17,0 \\
\hline Total & 86,2 & 13,8 \\
\hline
\end{tabular}

Si analizamos el tipo de ocupación (tabla 5), observamos entre los graduados en Información y Documentación un menor porcentaje de técnicos y profesionales y un mayor número de administrativos y empleados de oficina que entre el resto de los titulados.

Tabla 5. Ocupación en 2019 de los graduados universitarios del curso 2013-2014 que trabajaban en el momento de realización de la encuesta (\%).

\begin{tabular}{|l|c|c|c|c|c|c|}
\hline & Directores & $\begin{array}{c}\text { Técnicos y } \\
\text { profesionales } \\
\text { científicos e } \\
\text { intelectuales }\end{array}$ & $\begin{array}{c}\text { Técnicos pro- } \\
\text { fesionales de } \\
\text { apoyo }\end{array}$ & $\begin{array}{c}\text { Empleados con- } \\
\text { tables, adminis- } \\
\text { trativos y otros } \\
\text { empleados de } \\
\text { oficina }\end{array}$ & $\begin{array}{c}\text { Trabajadores de los } \\
\text { servicios de restau- } \\
\text { ración, personales, } \\
\text { protección y vende- } \\
\text { dores }\end{array}$ & $\begin{array}{c}\text { Otros } \\
\text { ofontes }\end{array}$ \\
\hline $\begin{array}{l}\text { Información y } \\
\text { Documentación }\end{array}$ & 5,5 & 39,0 & 16,1 & 25,2 & 7,6 & 6,7 \\
\hline $\begin{array}{l}\text { Ciencias Sociales, } \\
\text { Periodismo y } \\
\text { Documentación }\end{array}$ & 6,9 & 53,3 & 11,9 & 15,5 & 9,3 & 3,0 \\
\hline Total & 4,8 & 61,8 & 13,1 & 10,5 & & 6,5 \\
\hline
\end{tabular}

La Administración Pública continúa siendo el ámbito que proporciona mayores oportunidades de ocupación a los graduados en Información y Documentación (Moreiro-González, 2020), acogiendo a cerca de una cuarta parte de aquellos que trabajan (tabla 6). Si bien hay porcentajes significativos de titulados que trabajan en empresas e instituciones vinculadas al ámbito técnico y científico, queda un amplio margen de crecimiento en terrenos como las actividades sanitarias, transportes o comunicaciones en los que los graduados pueden desempeñar tareas de interés en la gestión documental y de datos (Abadal; Rubió, 2017). 
Tabla 6. Actividad económica principal de la empresa en la que trabajaban los graduados universitarios en Información y Documentación del curso 2013-2014.

\begin{tabular}{|c|c|}
\hline Actividad económica & $\%$ \\
\hline Administración Pública y defensa; Seguridad Social obligatoria & 23,5 \\
\hline Actividades profesionales, científicas y técnicas & 18,0 \\
\hline Actividades administrativas y servicios auxiliares & 13,9 \\
\hline Educación & 13,5 \\
\hline Actividades artísticas, recreativas y de entretenimiento & 5,7 \\
\hline Actividades sanitarias y de servicios sociales & 4,7 \\
\hline Hostelería & 4,6 \\
\hline Comercio al por mayor y al por menor reparación de vehículos de motor y motocicletas & 3,8 \\
\hline Transporte, almacenamiento & 3,1 \\
\hline Otros servicios & 3,1 \\
\hline Información y comunicaciones & 2,6 \\
\hline Actividades financieras y de seguros & 1,4 \\
\hline Industria manufacturera & 1,2 \\
\hline Construcción e ingeniería civil & 0,7 \\
\hline
\end{tabular}

De los resultados de la encuesta se desprende que, en al menos un $57 \%$ de los casos, el nivel de formación recibido por los graduados en Información y Documentación se adecúa a las necesidades de su puesto de trabajo (tabla 7). No obstante, en el extremo contrario, un $35 \%$ de los graduados afirma estar ocupando puestos para los que no es necesaria la titulación, siendo suficiente formación secundaria e, incluso, primaria. Esta cifra es sensiblemente superior a la que se observa entre el resto de los graduados en Ciencias Sociales (26\%) y entre el conjunto de graduados universitarios (20\%).

Tabla 7. Nivel de formación más apropiado para realizar el trabajo de los graduados universitarios del curso $2013-$ 2014 que trabajaban en el momento de realización de la encuesta (\%).

\begin{tabular}{|l|c|c|c|c|c|c|c|}
\hline & Doctorado & Máster & $\begin{array}{c}\text { Título univer- } \\
\text { sitario (excep- } \\
\text { to doctorado } \\
\text { o máster) }\end{array}$ & $\begin{array}{c}\text { Formación } \\
\text { profesional } \\
\text { de grado } \\
\text { superior }\end{array}$ & $\begin{array}{c}\text { Formación } \\
\text { profesional de } \\
\text { grado medio I } \\
\text { bachillerato }\end{array}$ & $\begin{array}{c}\text { Formación } \\
\text { profesional } \\
\text { básica }\end{array}$ & $\begin{array}{c}\text { ESO, } \\
\text { EGB, etc. }\end{array}$ \\
\hline $\begin{array}{l}\text { Información y } \\
\text { Documentación }\end{array}$ & 0,9 & 7,2 & 56,6 & 12,1 & 7,4 & 5,6 & 10,1 \\
\hline $\begin{array}{l}\text { Ciencias Sociales, } \\
\text { Periodismo y Doc- } \\
\text { umentación }\end{array}$ & 1,7 & 26,8 & 45,6 & 10,5 & 7,7 & 2,8 & 5,1 \\
\hline Total & 2,1 & 19,1 & 58,6 & 9,6 & 5,0 & 1,9 & 3,7 \\
\hline
\end{tabular}

Los resultados anteriores están directamente relacionados con los recogidos en la tabla 8, que muestra que un $22 \%$ de los graduados en Información y Documentación afirma ocupar un puesto de trabajo para el que no es necesaria formación en ninguna área concreta, lo que nos lleva a pensar que realizan tareas no especializadas. No obstante, en contraste con otros titulados en ciencias sociales, también es sensiblemente superior el porcentaje de graduados en Información y Documentación que considera que las tareas que realiza son exclusivas de su área de estudios.

Tabla 8. Área de estudios más apropiada para realizar el trabajo de los graduados universitarios del curso 2013-2014 que trabajaban en el momento de realización de la encuesta (\%).

\begin{tabular}{|l|c|c|c|c|}
\cline { 2 - 5 } & $\begin{array}{c}\text { Exclusivamente su } \\
\text { propia área de } \\
\text { estudios }\end{array}$ & $\begin{array}{c}\text { Su propia área de } \\
\text { estudios o alguna } \\
\text { relacionada }\end{array}$ & $\begin{array}{c}\text { Un área de estudios } \\
\text { totalmente diferente }\end{array}$ & $\begin{array}{c}\text { Ningún área de estu- } \\
\text { dios en particular }\end{array}$ \\
\hline $\begin{array}{l}\text { Información y Docu- } \\
\text { mentación }\end{array}$ & 28,6 & 28,7 & 20,5 & 22,2 \\
\hline $\begin{array}{l}\text { Ciencias Sociales, } \\
\text { Periodismo y Docu- } \\
\text { mentación }\end{array}$ & 15,5 & 48,0 & 22,0 & 14,5 \\
\hline Total & 25,2 & 50,4 & 14,2 & 10,3 \\
\hline
\end{tabular}


La tabla 9 evidencia la amplitud de la conocida brecha salarial de género. En el caso de los graduados en Información y Documentación, el $18 \%$ de los hombres afirma tener un salario superior a los 2.000 euros mensuales, mientras que únicamente el $4 \%$ de las mujeres declara superar esa cifra. Desequilibrios similares se observan en el área de ciencias sociales y entre el total de graduados universitarios. Por otro lado, los datos ponen de manifiesto los menores ingresos de los graduados en Información y Documentación en relación con los titulados en otras disciplinas. Mientras que únicamente el $8 \%$ de los graduados en Información y Documentación gana más de 2.000 euros mensuales, este porcentaje se eleva hasta el $19 \%$ entre los graduados en Ciencias Sociales y alcanza el $23 \%$ entre el total de graduados.

Tabla 9. Salario neto mensual de los graduados universitarios del curso 2013-2014 que trabajaban en el momento de realización de la encuesta (\%).

\begin{tabular}{|c|c|c|c|c|c|c|c|c|}
\hline & & 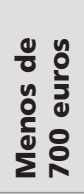 & 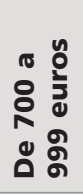 & 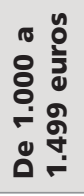 & 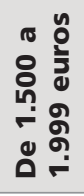 & 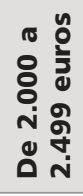 & 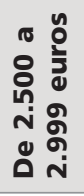 & 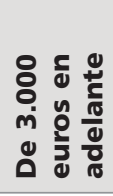 \\
\hline \multirow{3}{*}{$\begin{array}{l}\text { Información y } \\
\text { Documentación }\end{array}$} & Hombres & 10,9 & 6,2 & 49,9 & 15,4 & 9,1 & 0,0 & 8,6 \\
\hline & Mujeres & 11,0 & 20,2 & 49,4 & 15,5 & 1,5 & 2,4 & 0,0 \\
\hline & Total & 11,0 & 15,7 & 49,6 & 15,5 & 3,9 & 1,6 & 2,8 \\
\hline \multirow{3}{*}{$\begin{array}{l}\text { Ciencias Sociales, } \\
\text { Periodismo y } \\
\text { Documentación }\end{array}$} & Hombres & 5,9 & 8,7 & 33,4 & 23,0 & 16,4 & 5,6 & 7,0 \\
\hline & Mujeres & 9,8 & 13,7 & 43,4 & 20,3 & 8,8 & 2,5 & 1,5 \\
\hline & Total & 8,4 & 11,9 & 39,8 & 21,3 & 11,5 & 3,6 & 3,5 \\
\hline \multirow{3}{*}{ Total } & Hombres & 4,3 & 6,2 & 26,5 & 30,5 & 17,5 & 7,0 & 7,9 \\
\hline & Mujeres & 8,3 & 11,0 & 32,8 & 31,4 & 10,4 & 3,3 & 2,9 \\
\hline & Total & 6,6 & 9,0 & 30,1 & 31,0 & 13,4 & 4,9 & 5,0 \\
\hline
\end{tabular}

La inserción laboral y estabilización de los graduados en Información y Documentación parece relativamente rápida. De acuerdo con los datos de la tabla 10, el porcentaje de graduados que se mantuvo en el trabajo que ya realizaba en el momento de graduarse es muy superior al del resto de titulados.

Tabla 10. Tiempo transcurrido hasta encontrar el primer empleo de los graduados universitarios del curso 2013-2014 (\%).

\begin{tabular}{|c|c|c|c|c|c|c|c|}
\hline &  & 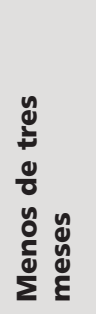 & 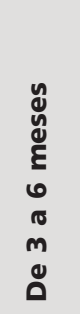 & 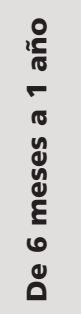 & 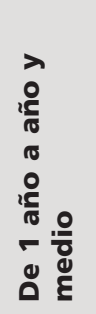 & 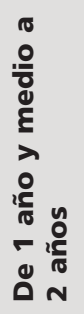 & 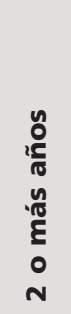 \\
\hline Información y documentación & 43,5 & 11,4 & 9,7 & 14,7 & 10,8 & 4,7 & 5,2 \\
\hline $\begin{array}{l}\text { Ciencias sociales, periodismo y } \\
\text { documentación }\end{array}$ & 29,8 & 18,0 & 10,6 & 11,0 & 10,8 & 7,3 & 12,6 \\
\hline Total & 28,1 & 22,9 & 11,2 & 11,9 & 9,9 & 5,7 & 10,2 \\
\hline
\end{tabular}

Para completar este apartado, cabe destacar que, en paralelo al trabajo del INE, el Ministerio de Ciencia, Innovación y Universidades publicó en 2019 el informe Inserción laboral de los egresados universitarios. Curso 2013-14. (Análisis hasta 2018) (MCIU, 2019). Este trabajo parte de dos fuentes de información complementarias:

- el Sistema Integrado de Información Universitaria (SIIU) que gestiona la Secretaría General de Universidades del Ministerio de Ciencia, Innovación y Universidades - que es también el punto de partida de la encuesta del INE-;

- la vida laboral de los afiliados a la Seguridad Social de la Tesorería de la Seguridad Social del Ministerio de Trabajo, Migraciones y Seguridad Social. 
La población objeto de estudio y el marco temporal son los mismos que en el trabajo del INE: los egresados universitarios del curso 2013-2014, de los que se analiza su inserción laboral en los cuatro años posteriores a la finalización de sus estudios a través, en este caso, de los datos que aporta la vida laboral de los afiliados a la Seguridad Social.

El informe del Ministerio incluye una tabla (páginas 109 y 110) con las bases de cotización de los egresados del curso 2013-2014 que contaban hasta 30 años de edad. En el caso de los graduados en Información y Documentación, la base de cotización era de 17.986 euros anuales, una cifra sensiblemente inferior a los 23.528 euros de media del conjunto de los graduados.

\section{Formación y aprendizaje}

La satisfacción con la titulación entre los graduados en Información y Documentación es ligeramente superior a la del conjunto de graduados en Ciencias Sociales y se sitúa al mismo nivel que entre el total de titulados universitarios (tabla 11). En consonancia con estos resultados, también son menos los graduados que afirman que realizarían otra carrera. No obstante, son algunos más los que declaran que no volverían a cursar estudios universitarios. Esta última afirmación tiene una cierta lógica si algunos de ellos están trabajando en tareas para las cuales la titulación no es necesaria o no está reconocida.

Tabla 11. Satisfacción con los estudios de los graduados universitarios del curso 2013-2014 (\%).

\begin{tabular}{|l|c|c|c|c|}
\cline { 2 - 5 } \multicolumn{1}{c|}{} & $\begin{array}{c}\text { Volvería a realizar } \\
\text { estudios } \\
\text { universitarios }\end{array}$ & $\begin{array}{c}\text { Volvería a realizar } \\
\text { estudios universita- } \\
\text { rios: volvería a realizar } \\
\text { su misma carrera }\end{array}$ & $\begin{array}{c}\text { Volvería a realizar } \\
\text { estudios universita- } \\
\text { rios: realizaria una } \\
\text { carrera distinta }\end{array}$ & $\begin{array}{c}\text { No volvería a realizar } \\
\text { estudios } \\
\text { universitarios }\end{array}$ \\
\hline $\begin{array}{l}\text { Información y } \\
\text { Documentación }\end{array}$ & 86,0 & 63,9 & 22,1 & 14,0 \\
\hline $\begin{array}{l}\text { Ciencias Sociales, } \\
\text { Periodismo y } \\
\text { Documentación }\end{array}$ & 86,7 & 57,5 & 29,3 & 13,3 \\
\hline Total & 87,9 & 63,7 & 24,2 & 12,1 \\
\hline
\end{tabular}

Si bien la encuesta no permite identificar en qué universidad han cursado los encuestados sus estudios, sorprende que el porcentaje de graduados de Información y Documentación que afirman haber realizado prácticas curriculares sea inferior al global (tabla 12). El Libro blanco aprobado en 2004 (Aneca, 2004) establecía como parte de los créditos obligatorios unas prácticas curriculares de 12 créditos. Es cierto que, posteriormente, las universidades han realizado cambios en sus planes de estudio (De-la-Moneda, 2018), pero una búsqueda en los planes actuales permite comprobar que las prácticas curriculares obligatorias siguen estando presentes en todos los planes de estudio a excepción de la Universitat Oberta de Catalunya (UOC), que contempla las prácticas profesionales como una asignatura optativa de 6 créditos.

En relación con las prácticas extracurriculares, los graduados de Información y Documentación afirman haber realizado menos prácticas de este tipo que los graduados del área de Ciencias Sociales, si bien ligeramente más que el total de graduados. Este dato podría estar relacionado con el hecho de que muchos de ellos trabajan durante los estudios, lo que les impide realizar prácticas no curriculares.

Tabla 12. Graduados universitarios en el curso 2013-2014 que han realizado prácticas en empresa mientras estudiaban (\%).

\begin{tabular}{|l|c|c|c|c|}
\cline { 2 - 5 } \multicolumn{1}{c|}{} & Realizaron prácticas & $\begin{array}{c}\text { Realizaron prácticas: } \\
\text { curriculares }\end{array}$ & $\begin{array}{c}\text { Realizaron prácticas: } \\
\text { extracurriculares }\end{array}$ & $\begin{array}{c}\text { No realizaron } \\
\text { prácticas }\end{array}$ \\
\hline $\begin{array}{l}\text { Información y } \\
\text { Documentación }\end{array}$ & 70,7 & 64,3 & 28,9 & 29,3 \\
\hline $\begin{array}{l}\text { Ciencias Sociales, } \\
\text { Periodismo y } \\
\text { Documentación }\end{array}$ & 79,1 & 68,5 & 33,0 & 20,9 \\
\hline Total & 74,5 & 61,3 & 27,9 & 25,5 \\
\hline
\end{tabular}

El alumnado de Información y Documentación se muestra poco propenso a la movilidad internacional (tabla 13). Resulta especialmente significativa la diferencia entre los graduados que realizaron una parte de sus estudios en el extranjero sin ninguna ayuda económica. Las becas son, si no determinantes, sí un elemento que favorece la realización de estancias fuera de España. 
Tabla 13. Graduados universitarios en el curso 2013-2014 según hayan realizado parte de sus estudios fuera de España (\%).

\begin{tabular}{|l|c|c|c|c|}
\cline { 2 - 5 } \multicolumn{1}{c|}{} & $\begin{array}{c}\text { Ha realizado parte } \\
\text { de sus estudios } \\
\text { fuera de España }\end{array}$ & $\begin{array}{c}\text { Ha realizado parte de } \\
\text { sus estudios fuera de } \\
\text { España: con una beca }\end{array}$ & $\begin{array}{c}\text { Ha realizado parte de } \\
\text { sus estudios fuera de } \\
\text { España: sin beca }\end{array}$ & $\begin{array}{c}\text { No ha realizado par- } \\
\text { te de sus estudios } \\
\text { fuera de España }\end{array}$ \\
\hline $\begin{array}{l}\text { Información y } \\
\text { Documentación }\end{array}$ & 6,2 & 6,0 & 0,2 & 93,8 \\
\hline $\begin{array}{l}\text { Ciencias Sociales, } \\
\text { Periodismo y } \\
\text { Documentación }\end{array}$ & 17,5 & 10,6 & 7,0 & 82,5 \\
\hline Total & 17,3 & 10,4 & 6,8 & 82,7 \\
\hline
\end{tabular}

Los bajos niveles de movilidad pueden estar relacionados con el menor conocimiento de otras lenguas, si bien es posible que los resultados de la tabla 14 estén condicionados por las lenguas propias de las comunidades autónomas. Sea como fuere, los graduados en Información y Documentación afirman conocer en menor medida que el resto de los graduados dos, tres o más idiomas.

Tabla 14. Graduados universitarios en el curso 2013-2014 según el número de idiomas que conoce distintos de su/s lengua/s materna/s (\%).

\begin{tabular}{|l|c|c|c|c|}
\cline { 2 - 5 } \multicolumn{1}{c|}{} & $\begin{array}{c}\text { No conoce otros } \\
\text { idiomas }\end{array}$ & Un idioma & Dos idiomas & $\begin{array}{c}\text { Tres o más } \\
\text { idiomas }\end{array}$ \\
\hline Información y Documentación & 3,8 & 59,9 & 27,0 & 9,3 \\
\hline Ciencias Sociales, Periodismo y Documentación & 4,5 & 50,2 & 33,7 & 11,6 \\
\hline Total & 4,5 & 51,0 & 33,4 & 11,2 \\
\hline
\end{tabular}

Finalmente, los graduados en Información y Documentación se manifiestan en un porcentaje elevado $(95 \%)$ como usuarios expertos o de nivel avanzado de las tecnologías de la información y la comunicación, más de siete puntos por encima de los graduados en ciencias sociales $(88 \%)$ y del total de graduados $(87 \%)$.

Tabla 15. Habilidades TIC de los graduados universitarios en el curso 2013-2014 (\%).

\begin{tabular}{|l|c|c|c|}
\cline { 2 - 4 } \multicolumn{1}{c|}{} & Usuario experto & $\begin{array}{c}\text { Usuario de nivel } \\
\text { avanzado }\end{array}$ & $\begin{array}{c}\text { Usuario de nivel } \\
\text { básico }\end{array}$ \\
\hline Información y Documentación & 19,7 & 75,5 & 4,7 \\
\hline Ciencias Sociales, Periodismo y Documentación & 14,1 & 74,0 & 12,0 \\
\hline Total & 20,1 & 67,3 & 12,6 \\
\hline
\end{tabular}

\section{Referencias}

Abadal, Ernest; Rubió, Anna (2017). “Evolución de los perfiles ocupacionales de los profesionales de la información". Anuario ThinkEPI, v. 11, pp. 58-62.

https://doi.org/10.3145/thinkepi.2017.06

Aneca (2004). Libro blanco Título de Grado en Información y Documentación. Madrid: Aneca. http://www.aneca.es/var/media/150424/libroblanco_jun05_documentacion.pdf

De-la-Moneda, Mercedes (2018). "Las cifras de la enseñanza universitaria en Documentación en España: 2016". Anuario ThinkEPI, v. 12, pp. 15-35.

https://doi.org/10.3145/thinkepi.2018.02

INE (2020). Encuesta de inserción laboral de titulados universitarios. Madrid: Instituto Nacional de Estadística. https://www.ine.es/dyngs//NEbase/es/operacion.htm?c=Estadistica_C\&cid=1254736176991\&menu=resultados\& $i$ $d p=1254735976597$

Ministerio de Ciencia, Innovación y Universidades (2019). Inserción laboral de los egresados universitarios. Curso $2013-$ 14. (Análisis hasta 2018). Madrid: Secretaría General Técnica del Ministerio de Ciencia, Innovación y Universidades. https://www.universidades.gob.es/stfls/universidades/Estadisticas/ficheros/Informe_de_Insercion_laboral_egresados.pdf

Moreiro-González, José-Antonio (2015). “¿Empiezan a cambiar las cosas en el mercado de trabajo de los titulados en información y documentación?". Anuario ThinkEPI, v. 10, pp. 49-55.

https://doi.org/10.3145/thinkepi.2016.04

Moreiro-González, José-Antonio (2020). "Un breve paseo estadístico por el empleo en el sector público, sin salir de casa". Anuario ThinkEPI, v. 14, e14a01.

https://doi.org/10.3145/thinkepi.2020.e14a01 